\title{
A Machine Vision System for Real-time Automated Gear Fatigue Pitting Detection
}

\author{
Zhang Jie \\ Mechatronics Center \\ Beijing Institute of \\ Technology Beijing, China \\ zhangjielovebit@126.com
}

\author{
Ma Shuyuan \\ Mechatronics Center \\ Beijing Institute of \\ Technology Beijing, China
}

\author{
Huang Jie \\ Mechatronics Center \\ Beijing Institute of \\ Technology Beijing, China
}

\author{
Long Zhenhai \\ Mechatronics Center \\ Beijing Institute of \\ Technology Beijing, China
}

\begin{abstract}
The machine vision system for the detection of the gear pitting is an essential element for the mechanical fatigue test, because the whole system effectively integrates internal information of the fatigue test to classify the related data into different sort automatically. Therefore, an industrial machine vision for gear pitting detection is proposed in this paper, and an image processing algorithm which mainly consists of image segmentation algorithm and contour computation is presented.
\end{abstract}

Keywords-machine vision, gear pitting, fatigue, image processing

\section{INTRODUCTION}

At present, most gear fatigue pitting detection and sorting operations are done by human vision. The main shortage of manual detect is low productivity, plenty of labors needed, poor accuracy and none useful data gained in the process. Nowadays industrial machine vision techniques, which have found a great increasing application on mechanical defect detection, have been increasingly crucial to keep the mechanical system gains in productivity [1]. The subject of machine vision is treated with emphasis of fundamental tools for image acquisition, processing and analysis.

The main task of the system is to inspect the amount and area of gear pitting on the tooth face. Moreover, this system provides a means of further classifying the pitting of gear in accordance with its size and specific positions. The experimental details, such as the test bench, working environment and some results are described. The image processing system to solve the problems, such as processing the large amount of image information and increasing the precision of detection is introduced in detail [2][3][4].

The machine vision system is presented for gear pitting detection in this paper based on real-time fatigue experiment. The work is aimed at:

- Building a machine vision model for gear pitting detection to describe its properties using image processing and hardware system.

- Get the quantitative data through the gear fatigue test, in order to optimize related design.

Sponsored by National Natural Science Foundation 50975030

\section{SYSTEM ARCHITECTURE}

The proposed machine vision system consists of three major components: gear contact fatigue test, image acquisition system and image processing system. Image processing system performs image segmentation, labeling and feature extraction.

\section{A. Experimental Environment}

A tooth surface fatigue machine is used to get the gear pitting. Enclosed structure is adopted towards the gear contact fatigue test bench.

In order to simulate the actual working environment, the test gear is working at a high speed under heavy load control. And the related parameters of gears are shown in Tab.1.

TABLE I. RELATED PARAMETERS OF GEARS

\begin{tabular}{|c|l|c|c|}
\hline \multirow{2}{*}{ NO. } & \multicolumn{3}{|c|}{ Related Parameters of Gears } \\
\cline { 2 - 4 } & \multicolumn{1}{|c|}{ Parameter Name } & Parameter & Value \\
\hline 1 & Teeth number of the active gear & $\mathrm{Z} 1$ & 26 \\
\hline 2 & Teeth number of the passive gear & $\mathrm{Z} 2$ & 27 \\
\hline 3 & Module of gear & $\mathrm{m}$ & 6 \\
\hline 4 & Tooth width & $\mathrm{b}$ & 40 \\
\hline
\end{tabular}

\section{B. Image Acquisition System}

The image acquisition system is used to capture the picture of the tooth surface the working gear for further processing. The main body of the system consists of the following parts: CCD camera, image card and illumination device. A CCD image sensor was clamped on a stand with lightning facilities. The image sensor captures images and sends them to the machine vision system.

Basler A602fc color camera together with the 1394 image card installed in the computer forms the sensor-digitizer combined with the computer. The LED lamps of 4 watt are served as the illumination. Also the light level can be regulated by adjusting the aperture of the microscope or adjusting the number of the lighting LED lights. The image captured by the camera is shown in Fig.1. 


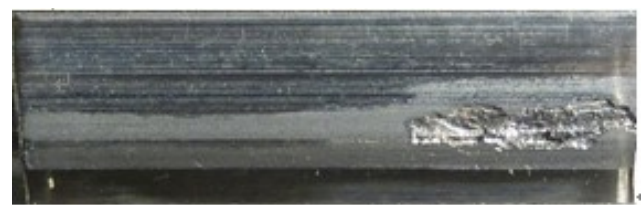

Figure 1 . The original image captured by camera

\section{Image Processing System}

Image processing system is used to change the input image to another image with the pitting characteristics required. In this system, highlight the details of the image, by regulating the signal-to-noise ratio and using image enhancement processing, in order to make the details to be easily observed. The first level image processing task includes noise removal, distortion compensation etc. Segmentation and labeling, feature extraction are generically classified as the intermediate level processing [6].

Now, the software realized in this paper and the details of the image processing algorithm is introduced shown in Fig.2.

In order to compare the difference of edge detection among the traditional algorithm and the improved algorithm presented for the fatigue pitting detection, the options of several algorithms are available. At the same time, three methods of noise reduction are optional in the system, which are threshold-value filter, average-filter and median filter respectively, so as to adapt to different kinds of images. The interface of the software system is shown in Fig.3 as follows.

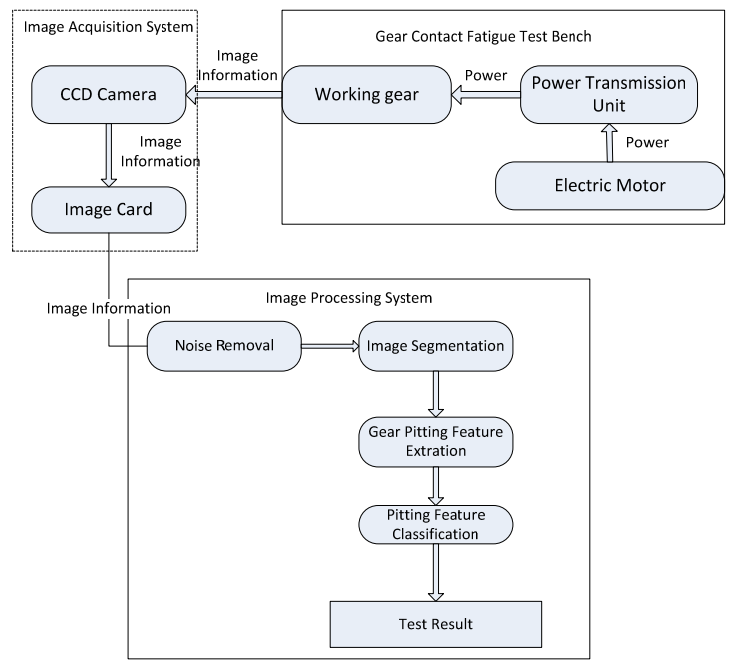

Figure 2. Block diagram of machine vision system

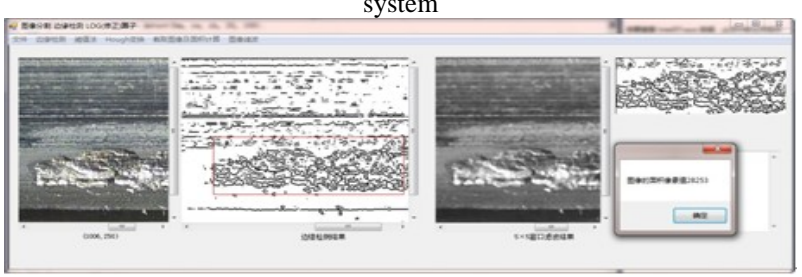

Figure 3. Interface of the Software System

\section{Gear Pitting Processing Algorithm}

\section{A. Noise Reduction Algorithm}

The image captured by CCD camera not only contains the edge information needed, but also includes the noise information of the experiment environment which will influence the accuracy of feature extraction [7]. As a result, noise reduction is especially essential before the edge detection. Nowadays there are three main means to realize this function: threshold-value filter, average-filter and median filter [8]

Noise reduction not only eliminates the noise of the image, but also wakens part of the useful information. As a nonlinear smooth filter based on sort statistical theory, it retains more effective information and reduces the noise better as well. After compare the different results of these three methods, as shown in Fig.4, a $5 \times 5$ window median filter is chosen in the end.

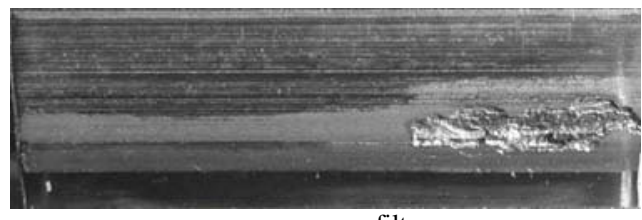

a. average-filter

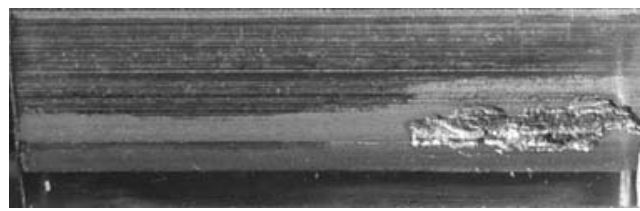

b. threshold-value filte

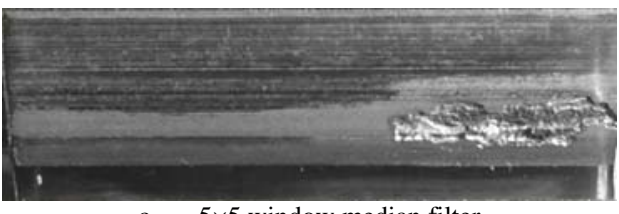

c. $5 \times 5$ window median filter

Figure 4. Different results of three noise filter

\section{B. Edge Detection Algorithm}

The contour of some designated area, as a basic characteristic, is widely used in the recognition, segmentation etc. Classic method based on gray of the gray grade of every pixel and the method using the multi-scale characteristic of the wavelet are commonly accepted. The main techniques are Laplace, Sobel, Canny and LOG (Laplace of Gaussian) algorithm etc [9]. Based on wavelet transform, Lipschitz exponent feature of the noise and signal are commonly applied as well [10]. With a view to the details of the objective image, a new segmentation algorithm which is realized based on LOG algorithm and Sobel algorithm is presented in this paper.

LOG, which is constituted of Gauss filter and Laplace edge detective operator, is proposed by Marr and Hildreth [11]. The output $\mathrm{h}(\mathrm{x}, \mathrm{y})$ of LOG operator is generated by Convolution computation:

$$
h(x, y)=\nabla^{2}[g(x, y) \bullet f(x, y)]
$$


On the basis of convolution computation, there is:

$$
h(x, y)=\nabla^{2}[g(x, y)] \bullet f(x, y)
$$

Where:

$$
\nabla^{2} g(x, y)=\left[\frac{x^{2}+y^{2}-2 \sigma^{2}}{\sigma^{4}}\right] e^{-\frac{x^{2}+y^{2}}{2 \sigma^{2}}}
$$

On the basis of LOG algorithm, the Sobel algorithm is incorporated with it. After LOG algorithm operation, the result becomes more reliable by applying $3 \times 3$ square window filtering of Sobel to the center point as shown in Fig.5. Two thresholds are put into use, a looser one to keep more edge information, and a strict one only to keep the edge information which is continuous with the edge detected by the looser threshold.

The original image is shown in Fig.1. After noise reduction and edge detection, the processing results are shown as Fig.6.

\begin{tabular}{|c|c|c|}
\hline 1 & & -1 \\
\hline 2 & & -2 \\
\hline 1 & & -1 \\
\hline
\end{tabular}

Figure 5. Sobel Algorithm
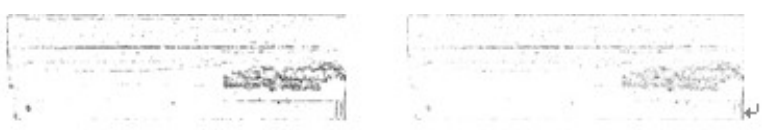

a.Kirsch

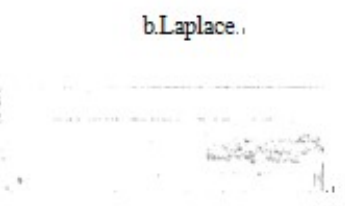

c.LOG

d.Prewitt.

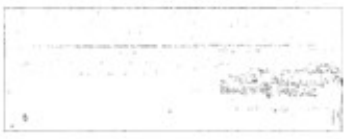

e.Roberts

f.Sobel.

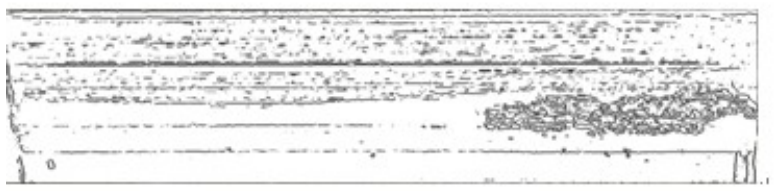

g.New Algorithm.

Figure 6. Test result of different edge detection algorithm

\section{Area Computation by Contour Tracing}

After denoising and edge detection, it still can't differentiate some edge information from noise. Therefore, the continuous boundary for area computation should be determined by taking out the separate points.

An improved eight-neighborhood contour tracing algorithm is presented in this paper. Freeman chain code uses 0,1,2,3,4,5,6,7 to stand for the eight directions, the improved algorithm sets point $\mathrm{P}(\mathrm{x}, \mathrm{y})$ for the current point, the directions of the pixels in the eight-neighborhood can be shown by the direction code in Fig.7.

This algorithm selects the upper left corner as the start point of the image, and scans the image from left to right and top to bottom. Starting from the first black point, as shown in Fig.5, the right direction of the point is defined as the initial search direction; if the point is black, and then takes it as the boundary point, otherwise revolve clockwise 45 degrees of the search direction until finding the first black point. Then take this black point as a new start one to cycle the above operation until the last black point return to the original point. Finally the contour of the area is gained for the further processing.

In this algorithm, 1,2,3 stand for the upward direction while 5,6,7 stand for the downward direction, and 3,4,5 stand for left when $0,1,7$ stand for right as well. It is easy to find the total number of each direction equals to the one of the opposite direction. Then, the equation of area computation is obtained as follow:

$$
\begin{gathered}
\text { Area }=x \cdot y=\operatorname{sum}(1,2,3) \cdot \operatorname{sum}(3,4,5) \\
\operatorname{sum}(1,2,3)=\operatorname{sum}(5,6,7) \\
\operatorname{sum}(3,4,5)=\operatorname{sum}(0,1,7)
\end{gathered}
$$

Finally, the pixels of each pitting region are calculated. The concave and convex parts are offset to each other, so as to ensure the accuracy of computation.

\begin{tabular}{|c|c|c|}
\hline $3 \nwarrow$ & $\uparrow 2$ & $\nearrow 1$ \\
\hline $4 \leftarrow$ & $\bullet$ & $\rightarrow 0$ \\
\hline $5 \swarrow$ & $\downarrow 6$ & $\searrow 7$ \\
\hline
\end{tabular}

Figure 7. Freeman Chain Code

\section{Pitting Classification}

According to the size characteristics, pattern recognition technology classifies the pitting into different categories: micropitting and macropitting. Save each region as an individual data, and sort them on the basis of the size, so as to confirm the specific circumstance of each pitting area. The threshold which differentiates these two conditions can be gained as appropriate.

Micropitting, which is grey or glitter in appearance by naked eyes, occurs in the dedendum, addendum, or both but seldom at the pitch line and is orienting against of tractive force. It is small in size within the limits of 5-10um in length, with the depth between 5-20um. On the contrary, macropitting occurs at the pitch line with large size. 


\section{RESULT}

As shown in Fig.6, the new edge detection algorithm improves the efficiency of region detection compared with the traditional algorithm. The new algorithm not only overcomes the LOG algorithm with a lot of noise in the image, but also overcomes shortcomings of the Sobel algorithm which lose amount of useful information. The new algorithm retains information of macropitting and micropitting, which demonstrate the details of pitting conditions.

Tab.2 shows the total count of pixels which stands for the pitting condition. It shows that with larger the number, the pitting shall be more serious. For the same image, the $5 \times 5$ median filter reduces more noise information than the other two methods with a smaller whole number of pixels. At the same time, the size of each pitting part is saved in PC as a separate data so that they can be classified. As explained before, the results also shows median filter can differential the useful image information from noise more effectively. With the total area of each surface in contact, the threshold for classification shall be determined more accurate by its location and size.

TABLE II. EXPERIMENTAL RESULTS OF DIFFERENT FILTERS

\begin{tabular}{|c|c|c|}
\hline \multirow{2}{*}{$\begin{array}{c}\text { Image } \\
\text { NO }\end{array}$} & \multicolumn{2}{|c|}{ Experimental results of different filters } \\
\hline & Filter & Area Value \\
\hline \multirow[t]{3}{*}{1} & threshold & 28469 \\
\hline & average & 26913 \\
\hline & median $5 \times 5$ & 25317 \\
\hline \multirow[t]{3}{*}{2} & threshold & 11485 \\
\hline & average & 12844 \\
\hline & median $5 \times 5$ & 11686 \\
\hline \multirow[t]{3}{*}{3} & threshold & 30786 \\
\hline & average & 31879 \\
\hline & median $5 \times 5$ & 28806 \\
\hline \multirow[t]{3}{*}{4} & threshold & 24343 \\
\hline & average & 24178 \\
\hline & median $5 \times 5$ & 20652 \\
\hline \multirow[t]{3}{*}{5} & threshold & 57926 \\
\hline & average & 60199 \\
\hline & median $5 \times 5$ & 64604 \\
\hline
\end{tabular}

\section{CONCLUSION AND FURTHER STUDIES}

In this work a novel machine vision system for gear fatigue pitting detection is presented. It has shown that the machine vision system in this paper is optimal in terms of efficiency and accuracy, compared with the manual detect. As the experimental results show, the algorithm presented a good performance in preserving small regions and edge locations when processing noisy images, as compared to the other methods. Nevertheless, with the future development of realtime image acquisition and algorithm optimization, the machine vision system for real-time detection shall be continually improved.

Actually this machine vision system not only needs to recognize the image in the computer, but also shall process the real-time image captured by the CCD camera, so as to get the conclusion required. The system consists of complex image processing and the process how the image is gained. Then in the future this system should be extended to set a coordinate system used to change the pixel to actual area of each part of pitting. At the same time, the algorithm shall be improved for better effect of detection.

\section{ACKNOWLEDGMENT}

The authors would like to thank the Key Laboratory of Fundamental Science for Advanced Machining for their support of this work. Special thanks to National Natural Science Foundation of China for their support under grants 50975030 .

\section{REFERENCES}

[1] ElMasry G, Wang N, Vigneault C, Qiao J, ElSayed A. Early Detection of Apple Bruises on Different Background Colors using Hyperspectral Imaging. LWT-Food Science and Technology, 2008, 41 (2): 337-345.

[2] Jin H. Study on the Machine Vision Based on Virtual Instrumentation. Computer Automated Measurement and Control, 2000, 8(1): 18-20.

[3] Hiroshi Sako, Recognition Strategies in Machine Vision Applications, International Machine Vision and Image Processing Conference, 2007.

[4] Sadayuki Tsugawa, Vision-Based Vehicles in Japan: Machine Vision Systems and Driving Control Systems, IEEE Transactions on Industrial Electronics, 1994, 41(4):398-405.

[5] A Guide for Fatigue Testing and the Statistical Analysis of Fatigue Data. ASTM STP9 1 A, 1964.

[6] Malik Abdul Waheed, Thornberg Benny, Meng Xiaozhou, Real-time Machine Vision System Using FPGA and Soft-core Processor, SPIE Conference on Real-Time Image and Video Processing, 2012.

[7] Loupas T, McDicken W N, Allan P L. An Adaptive Weighted Median Filter For Speckle Suppression In Medical Ultrasonic Images, IEEE Transactions on Circuits and Systems, 1989, 36( 1) : 129- 135

[8] A. P. Richard, A new algorithm for image noise reduction using mathematical morphology, IEEE Transaction on Image Processing, 1995 4(5):554-568.

[9] Paulo F U Gotardo, Olga R P Bellon, Luciano Silva, Range Image Segmentation By Surface Extraction Using An Improved Robust Estimator, IEEE Computer Society Conference on Computer Vision and Pattern Recognition, 2003.

[10] O Bellon, L Silva, New Improvements On Range Image Segmentation By Edge Detection, IEEE Signal Processing Letters, 2002, 9(2):43-45.

[11] D.Marr, Vision- A Computational Investigation into the Human Representation and Processing of Visual Information, Journal of Mathematical Psychology, 1982, 27(1):107-110. 